\title{
VCP/p97 in abnormal protein aggregates, cytoplasmic vacuoles, and cell death, phenotypes relevant to neurodegeneration
}

M Hirabayashi ${ }^{1,9}, \mathrm{~K}_{\text {Inoue }}{ }^{1,9}, \mathrm{~K}$ Tanaka ${ }^{1,9}, \mathrm{~K}$ Nakadate $^{2}$, Y Ohsawa ${ }^{3}$, Y Kamei ${ }^{1}$, AH Popiel ${ }^{1,4}$, A Sinohara ${ }^{5}$, A Iwamatsu ${ }^{5}$, Y Kimura ${ }^{6}$, Y Uchiyama ${ }^{3}$, S Hori ${ }^{1}$ and A Kakizuka*,1,7,8

1 The Fourth Department, Osaka Bioscience Institute, Osaka 565-0874, Japan

2 The Third Department, Osaka Bioscience Institute, Osaka 565-0874, Japan

${ }^{3}$ Department of Cell Biology and Neuroscience, Osaka University Graduate School of Medicine, Osaka 565-0871, Japan

${ }^{4}$ Graduate course, Kyoto University, Kyoto, Japan

${ }^{5}$ Central Laboratories for Key Technology, Kirin Brewery Co., Ltd., Kanagawa 236-0004, Japan

${ }^{6}$ Department of Tumor Cell Biology, The Tokyo Metropolitan Institute of Medical Science, Tokyo 113-8613, Japan

7 CREST, JST (Japan Science and Technology corporation)

${ }^{8}$ Current address: Kyoto University Graduate School of Biostudies, Kyoto 6068501, Japan

9 These authors contributed equally to this study

* Corresponding author: A Kakizuka, Kyoto University Graduate School of Biostudies, Kyoto 606-8501, Japan

Tel: +81-75-753-7675; Fax: +81-75-753-7676;

E-mail: kakizuka@lif.kyoto-u.ac.jp

Received 18.4.01; accepted 7.5.01

Edited by $\mathrm{H}$ Ichijo

\begin{abstract}
Neuronal cell death, abnormal protein aggregates, and cytoplasmic vacuolization are major pathologies observed in many neurodegenerative disorders such as the polyglutamine (polyQ) diseases, prion disease, Alzheimer disease, and the Lewy body diseases, suggesting common mechanisms underlying neurodegeneration. Here, we have identified VCP/ p97, a member of the AAA+ family of ATPase proteins, as a polyQ-interacting protein in vitro and in vivo, and report on its characterization. Endogenous VCP co-localized with expanded polyQ (ex-polyQ) aggregates in cultured cells expressing ex-polyQ, with nuclear inclusions in Huntington disease patient brains, and with Lewy bodies in patient samples. Moreover, the expression of VCP mutants with mutations in the 2nd ATP binding domain created cytoplasmic vacuoles, followed by cell death. Very similar vacuoles were also induced by ex-poly $Q$ expression or proteasome inhibitor treatment. These results suggest that VCP functions not only as a recognition factor for abnormally folded proteins but also as a pathological effector for several neurodegenerative phenotypes. VCP may thus be an ideal molecular target for the treatment of neurodegenerative disorders. Cell Death and Differentiation (2001) 8, 977-984.
\end{abstract}

Keywords: polyglutamine; protein accumulation; vacuole formation; neurodegeneration; neuronal cell death

\section{Introduction}

A class of inherited neurodegenerative disorders, including Huntington disease (HD) and Machado-Joseph Disease (MJD), is now known to be caused by expanded CAG repeats encoding for polyglutamines in the responsible genes. ${ }^{1,2}$ Expanded polyglutamines (ex-polyQs), typically of more than 40 repeats, have been shown to possess an intrinsic ability to aggregate in a poly $Q$ length- and a poly $Q$ concentration-dependent manner. The ability of polyQs to induce neurodegeneration in mice, and cell death in cultured cells appear to be inseparable from their intrinsic ability to aggregate, although there has been a report that the aggregates themselves are not essential for pathogenesis. ${ }^{3}$ This class of neurodegenerative disorders has thus been collectively called the 'polyglutamine diseases'. Accordingly, nuclear and/or cytoplasmic ex-polyQ-containing aggregates or inclusions have been observed in neurons of patients suffering from essentially all the polyQ diseases. Accumulation of abnormal proteins has also been observed in various other human neurodegenerative disorders ${ }^{1}$ (e.g. prion disease, Alzheimer disease, amyotrophic lateral sclerosis, and the Lewy body diseases such as Parkinson disease and dementia with Lewy bodies). It is notable that cytoplasmic vacuolization is another hallmark which has long been observed in a broad range of neurodegenerations, as well as during aging; ${ }^{4}$ cytoplasmic vacuoles have been observed in the affected neurons of several polyQ diseases, ${ }^{5,6}$ Parkinson disease, ${ }^{7}$ prion disease, ${ }^{8}$ and Alzheimer disease. ${ }^{9}$ Very little is known however about these vacuoles at the molecular level.

Several experimental model systems have previously been developed for the molecular and genetical analyses of the accumulation of abnormal proteins, and for the analysis of its relationship to neurodegeneration. ${ }^{10-12}$ In yeast, the [PSI+] element is inherited by a prion-like mechanism involving self-propagating Sup35p aggregates. ${ }^{13}$ It has been shown that the propagation of not only Sup35p, but also that of polyglutamine aggregates depends on the quantity of the chaperon Hsp104p (heat shock protein 104); both an increase and a decrease of Hsp104p levels decreases the formation of both types of aggregates. ${ }^{14-16}$ Hsp104p has two ATPase domains, and belongs to a class of the AAA (ATPases associated with diverse cellular activities) ATPase protein family with a hexameric ring structure (AAA+ class), which also includes NSF (Nethylmaleimide-sensitive fusion protein), VCP (valosincontaining protein)/p97/Cdc48p (cell division cycle protein 48), etc. ${ }^{17}$ A mammalian counterpart of Hsp104p however 
has not yet been identified. Here we report that VCP, a member of the AAA+ class of ATPases is an effector of several neurodegenerative phenotypes in ex-polyQ-expressing neuronal cells.

\section{Results}

During the search for molecules in mammalian cells which interact with ex-polyQs, all mammalian cultured cells examined were found to contain a protein with a molecular weight of approximately $100 \mathrm{kDa}$ which interacted with the glutathione S-transferase-fused MJD protein containing a 79 polyglutamine repeat, GST-MJD79 ${ }^{18}$ much more strongly than that fused with the MJD protein containing a 35 polyglutamine repeat, GST-MJD35. ${ }^{18}$ This protein was then purified from both COS cells and HeLa cells by GST-MJD79mediated affinity purification. Mass spectrometry and microsequencing analyses revealed that the protein from both cell types was VCP, a member of the AAA+ class of ATPases.
Northern blot analyses of human RNA showed that VCP was expressed ubiquitously in all tissues (not shown) and throughout the brain (Figure 1A), suggesting the possibility that VCP could be involved in human neurodegenerations occurring in all brain areas.

In vitro pull-down assays were performed in order to examine whether cloned VCP binds to MJD79. VCP indeed bound strongly to MJD79 and weakly to MJD35 (Figure 1B). Deletion analyses of MJD79 indicated that VCP binds directly to the stretch of ex-polyQs in the MJD protein (Figure 1C). Deletion analyses of VCP revealed that the portion containing amino acid residues 143-199, located towards the $\mathrm{N}$-terminus, is responsible for its binding to MJD79 (Figure 1D). We have tried several different experiments to test whether VCP acts as a chaperon towards the ex-polyQs, but have not been able to reach any convincing conclusions as yet. We have however observed that like Hsp104p, ${ }^{19}$ VCP can also bind to Hsp70 and Hsp40 (data not shown).
A

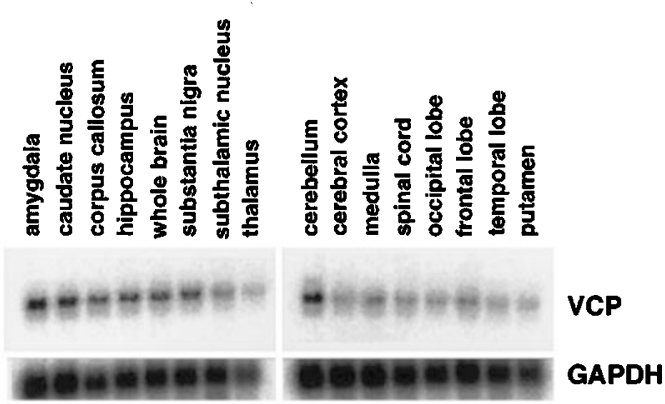

C

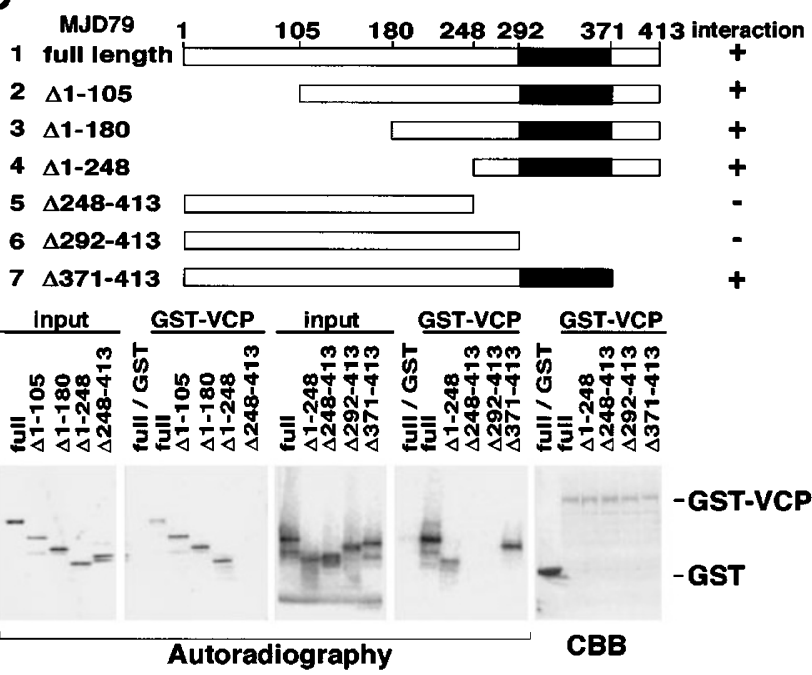

B

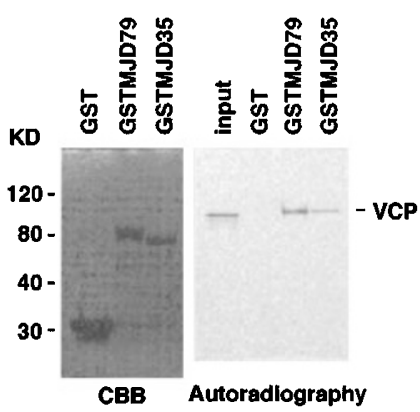

D

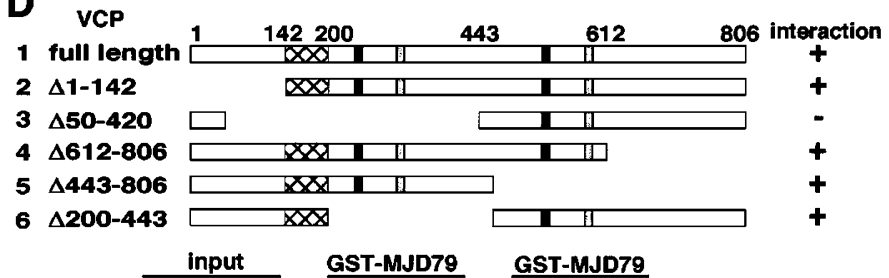

Figure 1 Identification of VCP as a protein interacting with the MJD protein containing an expanded polyglutamine repeat. (A) VCP and control GAPDH mRNA expression in 16 different areas of the human brain was analyzed by Northern blot analysis on a membrane preblotted by Clontech. (B) In vitro synthesized ${ }^{35}$ Slabeled VCP shows stronger interaction with GST-MJD79 than with GST-MJD35, and no interaction with GST. (C) Identification of the domain of MJD79 essential for its interaction with VCP via the GST pull-down assay. The illustrations show schematic structures of the MJD79 deletions. The polyglutamine stretches are indicates as black boxes. (D) Identification of the domain of VCP essential for its interaction with MJD79 via the GST pull-down assay. The illustrations show schematic structures of VCP deletions. The Walker A and Walker B motifs of the ATP binding domains in VCP are indicated as black and gray boxes, respectively. The crosshatched areas highlight the domain of VCP needed for its interaction with MJD79. Autoradiographs show $20 \%$ of the total amount of ${ }^{35}$ S-labeled proteins used for the GST pull-down assay (input) and the pulled down proteins (GST-VCP, GST-MJD79). CBB staining of the GST-fused proteins used are also presented (CBB) 
Consistent with the in vitro binding data shown above, endogenous VCP was found to colocalize with ex-polyQ stretches. Overexpressed flag-tagged 79 polyglutamine repeat fragments, Q79, were found to exist as aggregates both in the nucleus and in the cytoplasm in neuronally differentiated PC12 cells, ${ }^{20}$ and to colocalize with endogenous VCP (Figure 2A,B). In contrast, normal length polyQs such as Q14 and Q26 showed little binding to VCP (not shown), and remained in the cytoplasm without forming aggregates when expressed in neuronal PC12 cells. ${ }^{20}$ Most of the Q79-expressing cells were found to contain cytoplasmic vacuoles (Figure 2B). In brain sections from $\mathrm{HD}$ and MJD, VCP appeared to exist in the nuclear inclusions (Figure $2 \mathrm{C}$, and not shown). Furthermore, VCPpositive staining was observed in Lewy bodies (Figure 2D). The accumulation of VCP not only in the nuclear inclusions observed in the polyglutamine diseases but also in Lewy bodies indicate that VCP can recognize a broad range of abnormally folded proteins.

A series of VCP point mutants were created covering the different portions to test the possibility of generating mutants which may mimic ex-polyQ-induced phenotypes in cultured cells. Among the many mutants (e.g. K251A, E305Q, L306P, Y495A, Y495E, K524A, E578Q, L579P, $\mathrm{Y} 805 \mathrm{~A}$, and $\mathrm{Y} 805 \mathrm{E}$ ), only the $\mathrm{K} 524 \mathrm{~A}$ mutant with a substitution in the Walker A motif of the 2nd ATP binding domain clearly induced large cytoplasmic vacuoles in cells transfected with this mutant (Figure $3 \mathrm{~A}$ ). A deletion mutant of this Walker A motif $(\Delta 517-525)$ also formed large vacuoles when transfected into cells (data not shown). Furthermore, cells transfected with $\operatorname{VCP}(\mathrm{K} 524 \mathrm{~A})$ and $\operatorname{VCP}(\Delta 517-525)$ eventually died following vacuole formation. $N S F(K 549 A)$, a NSF mutant containing an equivalent mutation to $\operatorname{VCP}(\mathrm{K} 524 \mathrm{~A})$, has been reported to lose its ATPase activity and ATP binding, and has been proposed to function in a dominant-negative manner within the NSF hexameric complex. ${ }^{21}$ The predicted structure of NSF indicates that the $\mathrm{N}$-terminal portion is juxtaposed to the second ATPase domain. ${ }^{22,23}$ The binding of certain abnormally folded proteins to the $\mathrm{N}$-terminal portion of VCP may thus affect its ATPase activity and function similarly to $\operatorname{VCP}(\mathrm{K} 524 \mathrm{~A})$ and $\operatorname{VCP}(\Delta 517-525)$. NSF(K549A) however, was not able to induce such vacuoles or cell death (data not shown). Both vacuole formation and cell death induced by $\operatorname{VCP}(\mathrm{K} 524 \mathrm{~A})$ was suppressed by the co-expression of wild-type VCP in a dose-dependent manner (Figure 3B,C).

We previously observed that proteasome inhibitors such as MG115 and PSI caused the creation of numerous vacuoles in neuronal PC12 cells and HeLA cells, similar to those observed in $\operatorname{VCP}(\mathrm{K} 524 \mathrm{~A})$-expressing cells (Figure $4 \mathrm{~A})$. The proteasome is a complex that mediates the degradation of unnecessary proteins within cells, misfolded proteins being the obvious target. Treatment of cells with proteasome inhibitors has been shown to raise the amount of misfolded proteins within cells, ${ }^{24}$ which is similar to the situation in degenerating neurons containing abnormally folded proteins. Indeed, PC12 cells treated with such proteasome inhibitors showed the accumulation of abnormal protein aggregates, and these aggregates were clearly stained with the anti-VCP antibody (Figure 4A). Overexpression of VCP suppressed the formation of vacuoles created by such proteasome inhibitors transiently, but not permanently (Figure 4B,C).

We analyzed cells expressing ex-polyQ or VCP(K524A) by electron microscopy. Both types of cells contained many morphologically similar vacuoles. Typical vacuoles observed in both types of cells had ribosome-positive membranes (Figure 5A,D). Considering the evidence that VCP is involved in vesicle trafficking from the endoplasmic reticulum (ER) to the Golgi, these vacuoles are likely to be a result of abnormal budding and enlargement of the ER. Some vacuoles appeared to be connected to the tubular ER (Figure 5B). Note that very few ribosomes were observed on the membranes of greatly enlarged vacuoles observed in the late states of ex-polyQ-expressing cells (Figure 5B). Detailed morphological analyses of the processes of these vacuole formations will be reported elsewhere. The accumulation of enlarged ER-like vacuoles morphologically similar to those observed in cells expressing $\operatorname{VCP}(\mathrm{K} 524 \mathrm{~A})$ have been observed in the motor neurons of ALS model mice expressing mutant SODs. ${ }^{25}$ These results strongly indicate that VCP itself is involved in vacuole formation in cells containing ex-polyQs and other misfolded proteins as well. VCP thus should be reabbreviated for 'vacuole-creating protein'.

\section{Discussion}

In this study, we have identified VCP, a member of the AAA+ class of ATPases, as an MJD79-interacting protein by a biochemical approach. We have also identified VCP as a modifier of polyglutamine-mediated eye degeneration in Drosophila via genetic screening. Our genetic study using Drosophila identified ter94/VCP as a factor that worsens expolyQ-induced eye degeneration, indicating that VCP functions as an effector (manuscript in preparation). Consistently, in this present study, several lines of evidence demonstrate that VCP functions as an effector for several neurodegenerative phenotypes observed in cultured polyQ-expressing neuronal cells, namely vacuole creation and cell death.

Various similarities can be noted regarding structurefunction relationships within the AAA+ class of ATPases. The N-terminal portion of NSF is involved in protein binding, and binds to SNAP (soluble NSF attachment protein) via its $\mathrm{N}$-terminus. ${ }^{26}$ Likewise, the portion of VCP close to its $\mathrm{N}$-terminus is indispensable for its interaction with MJD79. Hsp104p has been reported to interact with Sup35p as well as mammalian $\operatorname{Pr}^{\mathrm{sc}}$, and $\mathrm{A} \beta$, although the precise domains of interaction have not been identified. ${ }^{27}$ We found that VCP was also able to bind to an intracellular portion of APP (amyloid precursor protein) containing $\mathrm{A} \beta$, as well as to $\mathrm{Hsp} 40$ and $\mathrm{Hsp} 70$. These observations consistently support the idea that Hsp104p and VCP have the intrinsic ability to act as a sensor of abnormally folded proteins, probably using portions near their $\mathrm{N}$-terminus to sense misfolded proteins either directly or indirectly via other proteins such as Hsp40 and Hsp70.

A hexameric structure such as that of VCP has the theoretical advantage to function as a system for 


\section{A PC12}
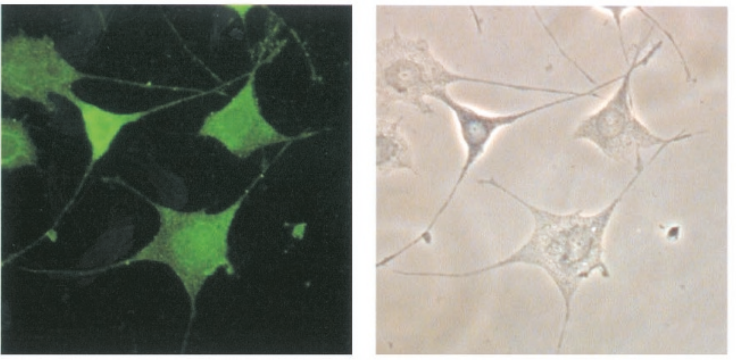

\section{B PC12-Q79 (Q79 induciton for $48 \mathrm{~h})$}
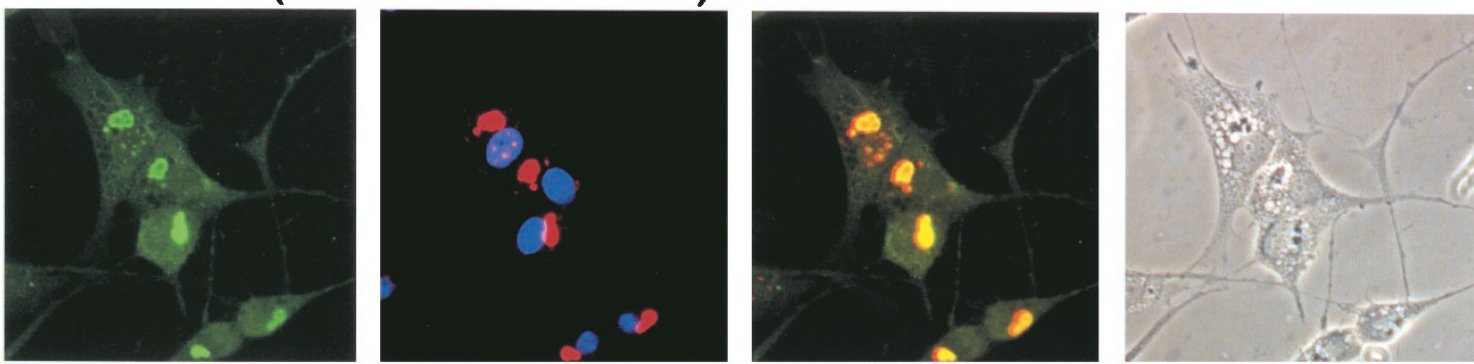

PC12-Q79 (Q79 induction for 96h)
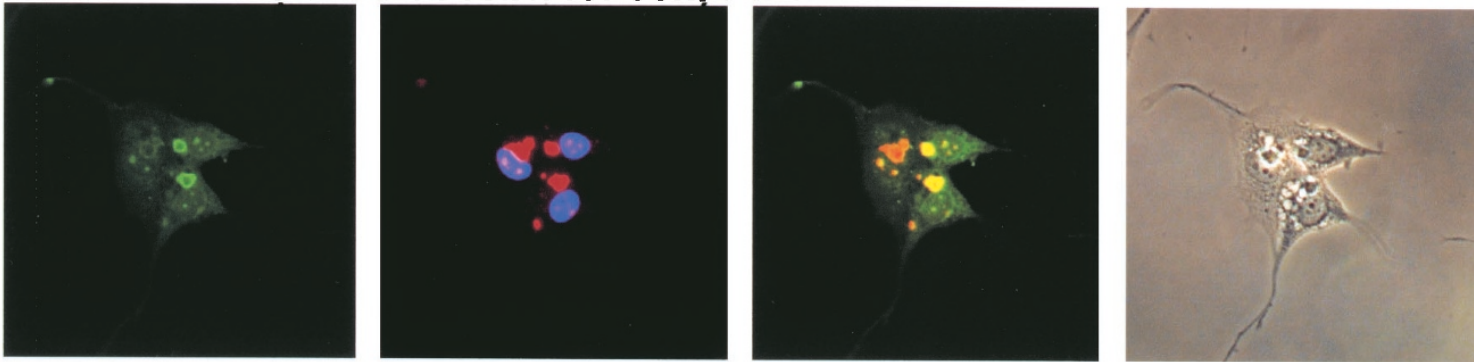

\section{Anti-VCP antibody Anti-flag ant}

Anti-VCP antibody and anti-flag antibody

C

D
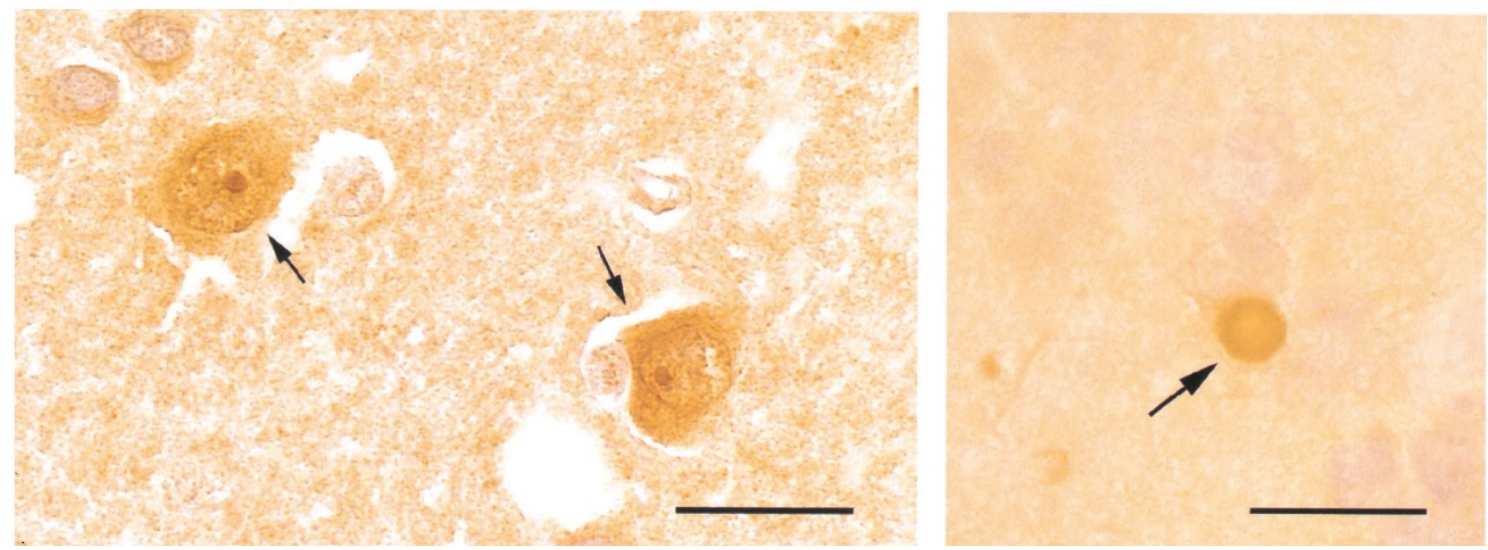

Figure 2 Co-localization of endogenous VCP with polyglutamine aggregates in PC12-Q79 cells, with nuclear inclusions in Huntington disease (HD), and with Lewy bodies in dementia with Lewy bodies (DLB). Endogenous VCP protein in PC12 cells (A) and PC12-Q79 cells (B) was stained with an anti-VCP antibody and visualized by FITC signals. Endogenous VCP was detected throughout the PC12 cells (A). Expressed Q79 was stained with an anti-flag antibody and visualized by Texas Red signals in PC12-Q79 cells, 48 h (upper panels) and $96 \mathrm{~h}$ (lower panels) after the induction of Q79. The yellow colors in the superimposed panels show the co-localization of endogenous VCP and Q79 (B). (C) Identification of endogenous VCP protein in nuclear inclusion-positive neurons from HD patients (arrows). Brain sections containing ubiquitin-positive nuclear inclusions from the postmortem cerebral cortex of Japanese patients with Huntington disease ${ }^{20}$ were stained with an anti-VCP antibody, and signals were visualized by the ABC method ${ }^{20}$ (brown signals). (D) Identification of endogenous VCP protein in Lewy bodies from 
monitoring the concentration of target molecules in cells; it should be able to recognize at least six different states by monitoring how many binding sites among the six are occupied by target molecules. Following this hypothetical step, Hsp104p functions as a chaperon to the misfolded proteins, whereas VCP appears to perform a toxic function similar to that induced by mutations in its second ATP binding domain, leading to vacuole forma- tion and eventually cell death. It is easily imaginable that such a toxic stoichiometry is increased in a certain agedependent frequency in neurons of patients suffering from polyglutamine diseases as well as other neurodegenerations with abnormally folded proteins, or even in aged brains. ${ }^{4}$ If so, the invention of small molecules which inhibit binding between VCP and abnormally folded proteins will be another strategy for the treatment or
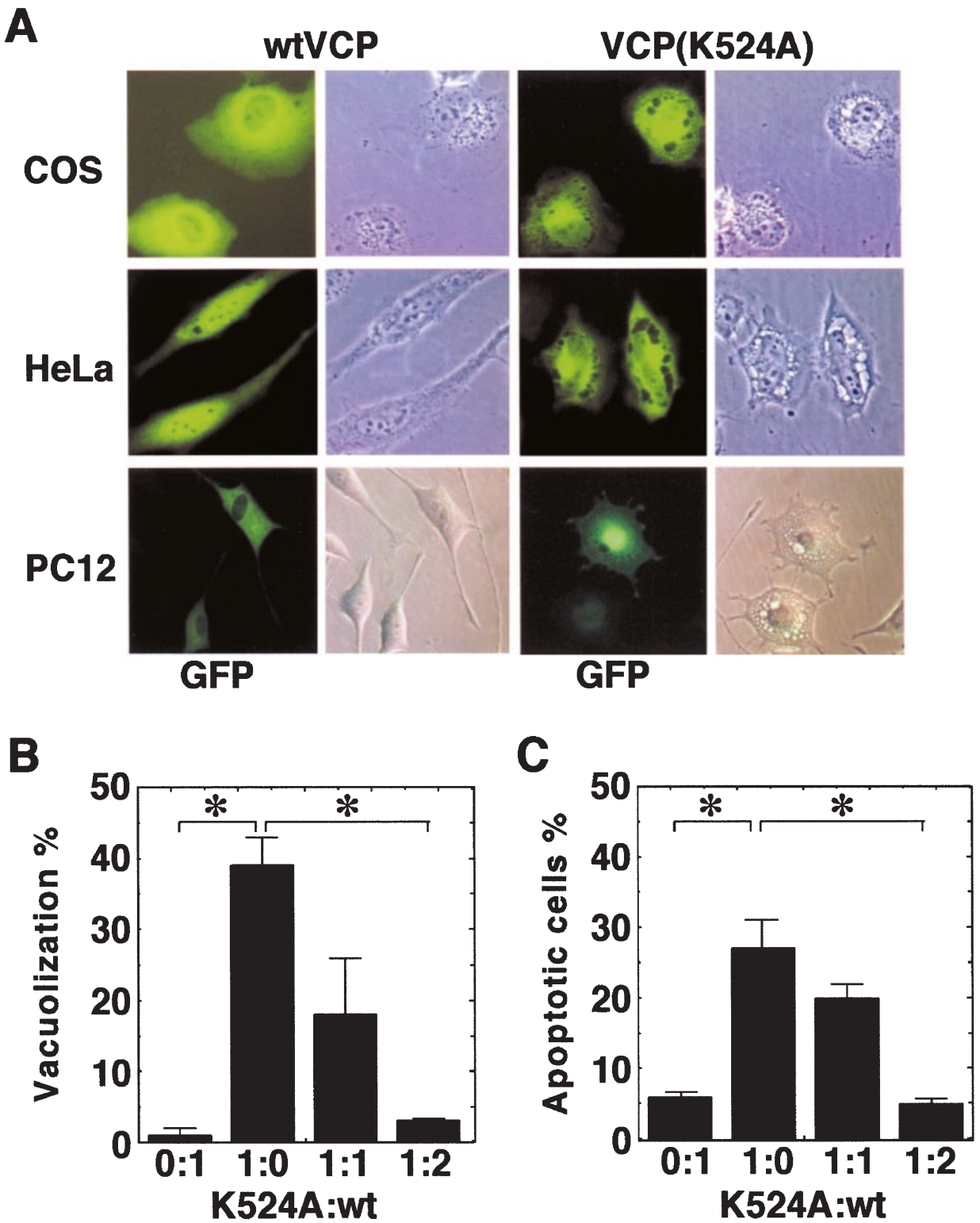

Figure 3 Vacuole formation caused by VCP(K524A) and its rescue by the expression of wild-type VCP. (A) Cytoplasmic vacuoles were induced by GFP-fused VCP(K524A) (right two panels) but not by GFP-fused wild-type VCP (left two panels) in COS, HeLa, and PC12 cells. (B, C) PC12 cells were co-transfected with VCP (K524A) in different ratios to wild-type VCP, and the percentages of cells containing vacuoles (B) and an apoptotic morphology (C) were examined $36 \mathrm{~h}$ after transfection

DLB patients (an arrow). Brain sections containing Lewy bodies from the postmortem brains of patients with DLB were stained with an anti-VCP antibody, and signals were visualized by the $\mathrm{ABC}$ method ${ }^{20}$ (brown signals). Bars represent $20 \mu \mathrm{m}$ (C, D) 
A
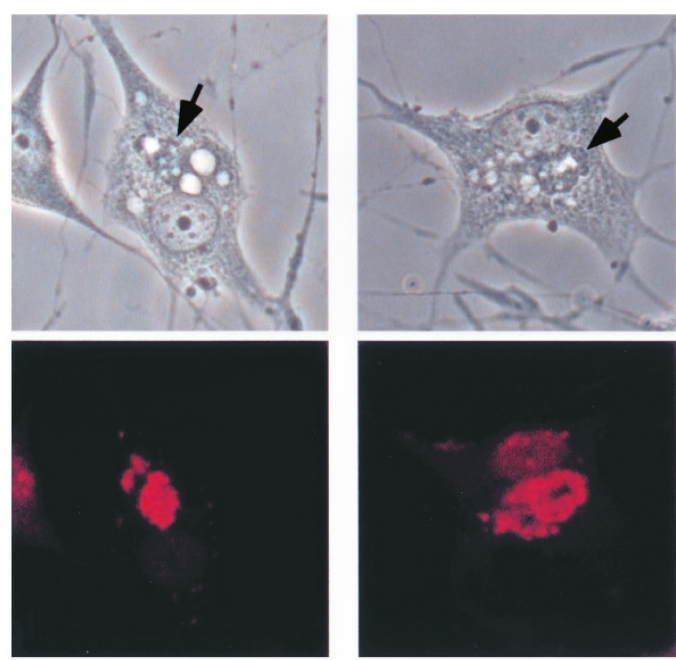
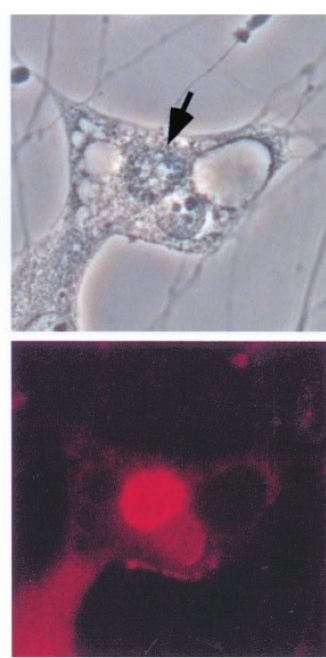

Anti-VCP antibody
B
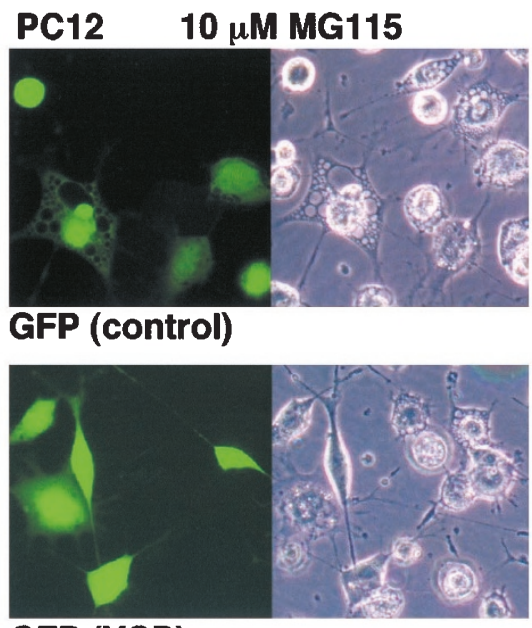

C

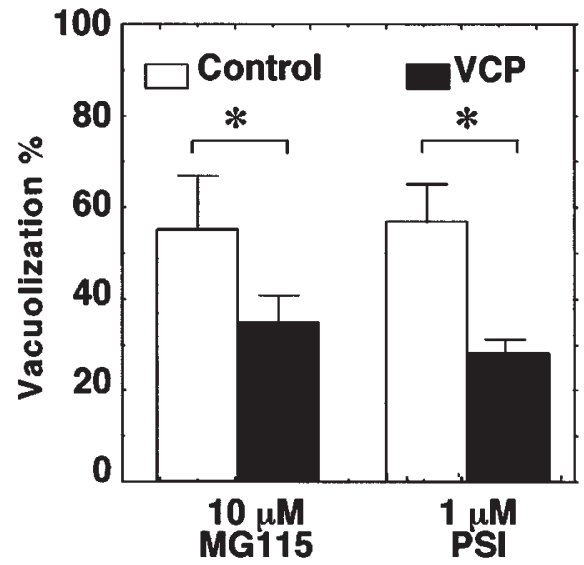

Figure 4 Proteasome treatment induces VCP-positive protein aggregates and large cytoplasmic vacuoles in PC12 cells. (A) Phase contrast views (upper panels) and immunohistochemical views (lower panels) of PC12 cells treated with $0.1 \mu \mathrm{M}$ PSI for $48 \mathrm{~h}$. Endogenous VCP protein was stained with an anti-VCP antibody and visualized by Texas Red signals (lower panels). Arrows indicate the protein aggregates observed in cells treated with proteasome inhibitors (upper panels). (B) Vacuole formation induced by $10 \mu \mathrm{M}$ MG115 was suppressed by the expression of wild-type VCP. (C) The percentages of cells containing vacuoles were examined in the control or wild-type VCP transfected PC12 cells $24 \mathrm{~h}$ after treatment with $10 \mu \mathrm{M}$ MG115 or $1 \mu \mathrm{M}$ PSI. Transfected cells were identified with GFP signals (GFP)

prevention of many types of neurodegenerations, as well as brain aging.

\section{Materials and Methods}

\section{GST pull-down assay}

A series of ${ }^{35} \mathrm{~S}$-labeled VCP or MJD proteins were prepared by the in vitro transcription and translation system (Promega). ${ }^{35} \mathrm{~S}$-labeled proteins were mixed with the GST-fusion proteins in NETN buffer with protease inhibitors (100 mM NaCl, $1 \mathrm{mM}$ EDTA, $20 \mathrm{mM}$ Tris $(\mathrm{pH} 7.4)$ $0.5 \% \mathrm{NP}-40,100 \mu \mathrm{g} \mathrm{ml}^{-1}$ aprotinin, $100 \mu \mathrm{g} \mathrm{ml}^{-1}$ leupeptin, $1 \mathrm{mM}$
PMSF) at $4^{\circ} \mathrm{C}$ with rotation for $2 \mathrm{~h}$, followed by centrifugation and washing with buffer $\mathrm{H}(20 \mathrm{mM}$ HEPES $(\mathrm{pH} 7.7), 50 \mathrm{mM} \mathrm{KCl}, 20 \%$ glycerol, $0.1 \% \mathrm{NP}-40$ ) repeated three times. Bound proteins were eluted from the beads with SDS sample buffer, boiled for $5 \mathrm{~min}$, resolved by SDS-PAGE, and detected by autoradiography.

\section{Cell lines, transfection and GFP assays}

Establishment and maintenance of PC12-Q79 cells that express Q79, a flag-tagged 79 repeat of polyglutamine from the MJD protein, under the control of the tet-off promoter has been described previously. ${ }^{20}$ For GFP assays, transfection was performed using Transfast (Promega); $5 \times 10^{4}$ cells in a 6 -well dish were transfected with $1 \mu \mathrm{g}$ pCAGGS 

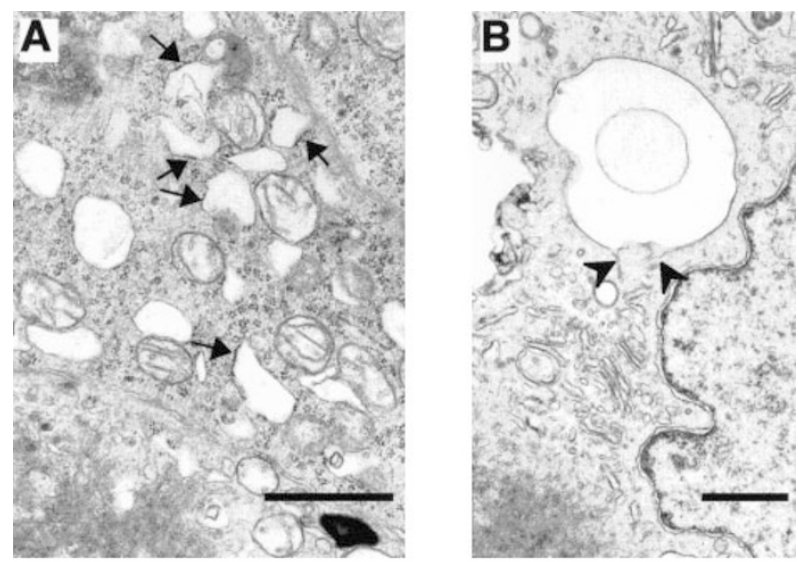

\section{Cell treatment for immunocytochemistry}

Cells were rinsed with PBS and fixed with $4 \%$ paraformaldehyde or $100 \%$ ethanol for $10 \mathrm{~min}$ at room temperature. After fixation, cells were permeabilized with $0.2 \%$ Triton $\mathrm{X}-100$ for $3 \mathrm{~min}$ and incubated at $4{ }^{\circ} \mathrm{C}$ overnight with M5, a mouse monoclonal anti-flag antibody (Kodak), and/or a rabbit polyclonal anti-VCP antibody. Subsequently, cells were treated with Texas-Red conjugated and/or FITC conjugated secondary antibodies (Jackson Immuno Research) and mounted in anti-fade solution with DAPI (Vector Laboratories).
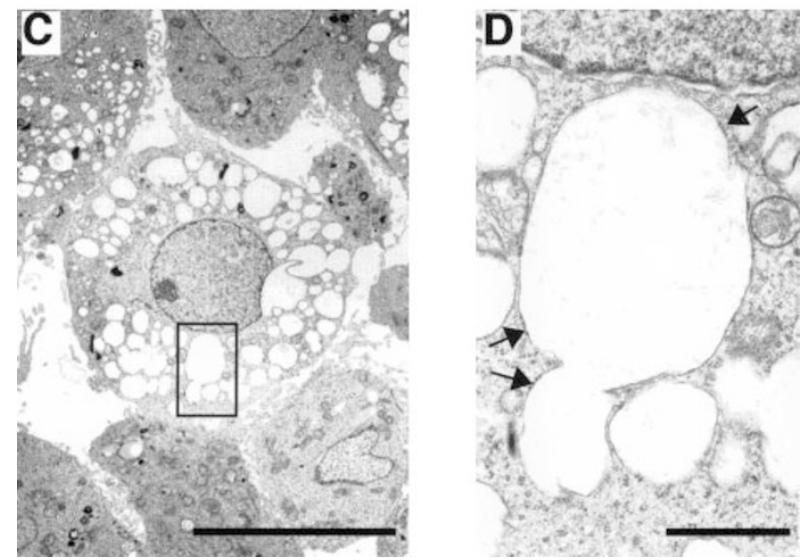

Figure 5 Ultrastructural analyses of the vacuoles induced by polyglutamines and $\operatorname{VCP}(\mathrm{K} 524 \mathrm{~A})$. (A-D) Electron micrograph of PC12 cells expressing Q79 $(\mathbf{A}, \mathbf{B})$ and $\operatorname{VCP}(\mathrm{K} 524 \mathrm{~A})(\mathbf{C}, \mathrm{D})$ under the control of the tet-off promoter. Cells were examined $24 \mathrm{~h}(\mathbf{A})$ and $48 \mathrm{~h}(\mathbf{B}-\mathbf{D})$ after removal of tetracycline. Bars represent $1 \mu \mathrm{m}(\mathbf{A}, \mathbf{B}, \mathbf{D})$ and $10 \mu \mathrm{m}(\mathbf{C})$; arrows, membrane portions with ribosomes; arrowheads, fused portions between ER and abnormally enlarged vacuoles. The area shown in (D) is an enlarged view of the rectangle in (C)

vector containing cDNAs together with $0.2 \mu \mathrm{g}$ pEGFP vector (Clontech). Twenty-four hours after transfection, tetracycline was removed from the medium, and an additional 48,72 or $96 \mathrm{~h}$ later, cell morphology and GFP signals were analyzed using a microscope. NGF was added to PC12 or PC12-Q79 cells at a concentration of $50 \mathrm{ng} \mathrm{ml}^{-1}$ at the time of tetracycline removal. The ratio of cells containing vacuoles and apoptotic features were determined by dividing the number of GFP-positive cells containing vacuoles or showing apoptotic morphologies (e.g. membrane blebbing and cell shrinkage) by the total number of GFP-positive cells (approximately 200 cells). In the Figures, the mean values of triplicate experiments are presented, and s.d. are included as bars. Statistic values from the Student $t$-test are also included; ${ }^{*} P<0.05 ;{ }^{\star *} P<0.01$.

\section{VCP antibodies}

The GST-VCP protein was expressed in E. Coli and thrombin-cleaved VCP protein was purified by FPLC (Pharmacia). Rabbits were immunized with the purified VCP protein following standard procedures. The resulting immune serum was affinity-purified against VCP proteins immobilized on AminoLink column (PIERCE).

\section{Acknowledgements}

We thank $H$ Yoshii and $M$ Sugimoto for secretarial assistance in the preparation of this manuscript. We thank G Sobue (Nagoya University) for postmortem brain samples with HD, and M Baba and T Iwatsubo (Tokyo University) for postmortem brain samples for DLB. This work was supported in part by research grants from the Ministry of Education, Science, Sports, and Culture of Japan, and the Ministry of Welfare of Japan, the Yamanouchi Foundation for Research on Metabolic Disorders, the Naito Foundation, and the Uehara Foundation.

\section{References}

1. Kakizuka A (1998) Protein precipitation: a common etiology in neurodegenerative disorders? Trends Genet. 14: 396-402

2. Price DL (1999) New order from neurological disorders. Nature 399: A3-5

3. Saudou F, Finkbeiner S, Devys D and Greenberg ME (1998) Huntingtin acts in the nucleus to induce apoptosis but death does not correlate with the formation of intranuclear inclusions. Cell 95: 55-66

4. Okamoto K, Hirai S, lizuka T, Yanagisawa T and Watanabe M (1991) Reexamination of granulovacuolar degeneration. Acta Neuropathol. 82: 340 345

5. Sapp E, Schwarz C, Chase K, Bhide PG, Young AB, Penney J, Vonsattel JP, Aronin N and DiFiglia M (1997) Huntingtin localization in brains of normal and Huntington's disease patients. Ann. Neurol. 42: 604-612

6. Klement IA, Skinner PJ, Kaytor MD, Yi H, Hersch SM, Clark HB, Zoghbi HY and Orr HT (1998) Ataxin-1 nuclear localization and aggregation: role in polyglutamine-induced disease in SCA1 transgenic mice. Cell 95: 41-53

7. Anglade P, Vyas S, Javoy-Agid F, Herrero MT, Michel PP, Marquez J, MouattPrigent A, Ruberg M, Hirsch EC and Agid Y (1997) Apoptosis and autophagy in nigral neurons of patients with Parkinson's disease. Histol. Histopathol. 12: 2531

8. Grigoriev V, Escaig-Haye F, Streichenberger N, Kopp N, Langeveld J, Brown P and Fournier JG (1999) Submicroscopic immunodetection of PrP in the brain of a patient with anew-variant of Creutzfeldt-Jakob disease. Neurosci. Lett. 264:57 60

9. Cataldo AM, Hamilton DJ, Barnett JL, Paskevich PA and Nixon RA (1996) Properties of the endosomal-lysosomal system in the human central nervous system: disturbances mark most neurons in populations at risk to degenerate in Alzheimer's disease. J. Neurosci. 16: 186-199

10. Kakizuka A (1997) Degenerative ataxias: genetics, pathogenesis and animal models. Curr. Opin. Neurol. 10: 285-290

11. Bates GP, Mangiarini $L$ and Davies SW (1998) Transgenic mice in the study of polyglutamine repeat expansion diseases. Brain Pathol. 8: 699-714

12. Fortini ME and Bonini NM (2000) Modeling human neurodegenerative diseases in Drosophila: on a wing and a prayer. Trends Genet. 16: 161-167

13. Kushnirov VV and Ter-Avanesyan MD (1998) Structure and replication of yeas prions. Cell 94: 13-16

14. Chernoff YO, Lindquist SL, Ono B, Inge-Vechtomov SG and Liebman SW (1995) Role of the chaperone protein $\mathrm{Hsp} 104$ in propagation of the yeast prion-like factor psi $^{+}$]. Science 268: $880-884$

15. Krobitsch S and Lindquist S (2000) Aggregation of huntingtin in yeast varies with the length of the polyglutamine expansion and the expression of chaperone proteins. Proc. Natl. Acad. Sci. USA 97: 1589-1594 
16. Satyal SH, Schmidt E, Kitagawa K, Sondheimer N, Lindquist S, Kramer JM and Morimoto RI (2000) Polyglutamine aggregates after protein folding homeostasis in Caenorhabditis elegans. Proc. Natl. Acad. Sci. USA 97: 5750-5755

17. Neuwald AF, Aravind L, Spouge JL and Koonin EV (1999) AAA+: A class of chaperone-like ATPases associated with the assembly, operation, and disassembly of protein complexes. Genome Res. 9: 27-43

18. Ikeda H, Yamaguchi M, Sugai S, Aze Y, Narumiya S and Kakizuka A (1996) Expanded polyglutamine in the Machado-Joseph disease protein induces cell death in vitro and in vivo. Nat. Genet. 13: 196-202

19. GloverJRand LindquistS (1998) Hsp104, Hsp70, and Hsp40: anovel chaperone system that rescues previously aggregated proteins. Cell 94: 73-82

20. Yasuda S, Inoue K, Hirabayashi M, Higashiyama H, Yamamoto $Y$, Fuyuhiro $H$ Komure O, Tanaka F, Sobue G, Tsuchiya K, Hamada K, Sasaki H, Takeda K Ichijo H and Kakizuka A (1999) Triggering of neuronal cell death by accumulation of activated SEK1 on nuclear polyglutamine aggregations in PML bodies. Genes Cells 4: 743-756

21. Whiteheart SW, Rossnagel K, Buhrow SA, Brunner M, Jaenicke R and Rothman JE (1994) $N$-ethylmaleimide-sensitive fusion protein: a trimeric ATPase whose hydrolysis of ATP is required for membrane fusion. J. Cell Biol. 126: 945-954
22. Hanson PI, Roth R, Morisaki H, Jahn R and Heuser JE (1997) Structure and conformational changes in NSF and its membrane receptor complexes visualized by quick-freeze/deep-etch electron microscopy. Cell 90: 523-535

23. May AP, Misura KM, Whiteheart SW and Weis WI (1999) Crystal structure of the amino-terminal domain of $\mathrm{N}$-ethylmaleimide-sensitive fusion protein. Nat. Cell Biol. 1: 175-182

24. Bush KT, Goldberg AL and Nigam SK (1997) Proteasome inhibition leads to a heat-shock response, induction of endoplasmic reticulum chaperones, and thermotolerance. J. Biol. Chem. 272: 9086-9092

25. Dal Canto MC and Gurney ME (1995) Neuropathological changes in two lines of mice carrying a transgene for mutant human $\mathrm{Cu}, \mathrm{Zn} \mathrm{SOD}$, and in mice overexpressing wild type human SOD: a model of familial amyotrophic lateral sclerosis (FALS). Brain Res. 676: 25-40

26. Nagiec EE, Bernstein A and Whiteheart SW (1995) Each domain of the $N$ ethylmaleimide-sensitive fusion protein contributes to its transport activity. J. Biol. Chem. 270: 29182-29188

27. Schirmer EC and Lindquist S (1997) Interactions of the chaperone Hsp104 with yeast Sup35 and mammalian PrP. Proc. Natl. Acad. Sci. USA 94: 13932-13937 